EPSC Abstracts

Vol. 15, EPSC2021-383, 2021

European Planetary Science Congress 2021

(C) Author(s) 2021. This work is distributed under

the Creative Commons Attribution 4.0 License.

\title{
GMAP - European Mapping efforts for Geologic Mapping of Planetary bodies
}

Andrea Nass ${ }^{1}$, Matteo Massironi ${ }^{2}$, Angelo Pio Rossi ${ }^{3}$, Luca Penasa ${ }^{2}$, Riccardo Pozzobon ${ }^{2}$, Carlos Brandt $^{3}$, Giacomo Nodjoumi ${ }^{3}$, Monica Pondrelli ${ }^{4}$, Marco Pantaloni ${ }^{5}$, Vallentina Galluzzi ${ }^{6}$, Francesca Altieri $^{6}$, Alessandro Frigeri ${ }^{6}$, Christian Carli ${ }^{6}$, Lorenza Giacomini ${ }^{6}$, Daniel Mège ${ }^{7}$, Joanna Gurgurewicz ${ }^{7}$, Pierre-Antoine Tesson ${ }^{7}$, Lucia Marinangeli ${ }^{8}$, Carolyn van der Bogert ${ }^{9}$, and Claudia Poehler ${ }^{9}$

${ }^{1}$ DLR / Institut of Planetary Research, Planetary Geology, Berlin, Germany (andrea.nass@dlr.de)

${ }^{2}$ Dipartimento di Geoscienze, Università di Padova

${ }^{3}$ Jacobs University Bremen

${ }^{4}$ IRSPS, Università G. d'Annunzio, Pescara

${ }^{5}$ Department of Geological Survey of Italy (ISPRA), Rome

${ }^{6}$ National Institute for Astrophysics (INAF), Rome

${ }^{7}$ Centrum Badań Kosmicznych Polskiej Akademii Nauk, Warsaw

${ }^{8}$ uNICH Università G. d'Annunzio, Chieti

${ }^{9}$ Institut für Planetologie, Westfälische Wilhelms-Universität Münster

Introduction: One essential part of NASA's planetary geologic mapping program [1] is to coordinate and standardize the geological map process and products in planetary science. This important role is taken by the Astrogeology Team as USGS since the early sixties.

Within the scope of an EU project called PLANetary MAPping (PLANMAP, [2]), which ended this year, initial steps to develop complementary expertise in the EU was done. To continue addressing the major scientific and technological challenges facing modern planetary science and strengthen Europe's position and the forefront of space exploration a new pan-EU infrastructure, the EUROPLANET 2024 Research Infrastructure (EPN-2024-RI), is coordinating mapping efforts in the EU and with international partners.

One component of this 4-year-project is the Geologic MApping of Planetary bodies (GMAP). This aims to serve the European planetary community through an infrastructure to foster, support, and sustain the production of planetary geological maps and related products following standard procedures [e.g., 3]). In order to do so, GMAP is directly building on the PLANMAP work [2], and several partners and institutions with previous experience in planetary geologic mapping are involved. That means a planetary scientist can produce a geological map or a derived higher-level product through GMAP Virtual Access (VA) with the help and advice of the GMAP partner institutions, who will provide base-maps and technical aid as part of the Joint Research Activity (JRA). The maps will provide support for ongoing and future planetary missions, training activities, and non-standard science-driven mapping projects, such as space resource mapping.

GMAP - motivation and focus: The primary focus of GMAP is to streamline the processes which are involved in the production of geological and geomorphological maps of planetary surfaces. Here, we are mainly collecting existent approaches and related documents which handle the 
standardization of GIS-based mapping processes to enable the European community in creating cartographic products. The aim is to describe, develop, store, combine (!), access, update, revise, and finally, visualize scientific cartographic products. As soon as these steps can be handled in welldefined workflow and distributed among researchers and mappers, the highest possible level of homogenization, and thus standardization, is reached. This is the essential step to use these research products as a basis for broader studies. During the first year, coordination activities targeted the planning and the initial setup of digital infrastructure services that will be needed for supporting VA and JRA activities. The domain europlanet-gmap.eu was acquired by GMAP and will serve as the entry point for presenting the GMAP initiative, collecting most notable resources, for users' access, for providing basic guidance for publishing new maps, request support and contribute to the overall project. The website is built on the same open source Content Management System (WordPress, [4]) already employed for the main Europlanet website, on https://europlanetsociety.org. The GMAP data portal (see figure 1, [5]) and additional services and tools are being setup.

GMAP - requirements and developments: In order to extract the requirements to support the European community in streamlining their planetary geological maps, a document was produced during the last year of JRA activities. The document contains state of the art information in this field and addresses the geologic mapping and cartographic aspects of the various Solar System bodies.

Geologic process-specific and body-specific best practice and published case studies are included in [1]. The approaches for two-dimensional mapping and three-dimensional geologic mapping and modelling are introduced, as well as the range of non-standard map types that are envisaged within GMAP activities.

In particular the following main topics are in development: 1) a mapping guide with essential information for the GIS-based mapping process, including CRS symbology, metadata, and naming conventions; 2) mapping templates for GIS-based mapping and for final map layout, as well as instructions about naming conventions [6].

Mapping review directions are indicated, as well data sharing, distribution and discovery. Proposed standards, best practices, and tools are based on those existing, as well as on additional or new developments and adaptations [e.g. 7]. The document will be periodically updated.

GMAP - summary and outlook: The development of the GMAP data portal [5] was initiated, based on existing developments from PLANMAP. The availability of GMAP products and underlying datasets is going to be FAIR (findable, accessible, interoperable, and reusable [8]), as also recommended by the VA Review Board (see also [9]), and building on the practices of PLANMAP [10], see also e.g. [11]). The use of existing tools by NASA and USGS such as Integrated Software for Imagers and Spectrometers (ISIS, [12]), and Ames Stereo Pipeline (ASP, [13]) will be promoted. Moreover, in addition to the community support by the GMAP VA, interaction with the community via OpenPlanetary [14] is also planned. All further information and current developments are available via [15] and [16].

Acknowledgments: GMAP and Europlanet 2024 RI have received funding from the European Union's Horizon 2020 research and innovation programme under grant agreement No 871149.

$\begin{array}{lllll}\text { References: } & {[1]} & \text { Astrogeology am at }\end{array}$ USGS, https://www.usgs.gov/centers/astrogeology-science-center, [2] https://planmap.eu/, [3] Nass, et al., (2020) Standard definition Document 1st iteration, available online at https://www.europlanet-gmap.eu/about-gmap/deliverables, [4] https://de.wordpress.org/, [5] GMAP data portal, available online at https://data.europlanet-gmap.eu, [6] GMAP Consortium (2021) GMAP wiki documentation and service pages, available online at https://wiki.europlanetgmap.eu/, [7] Penasa, L., et al. (2020) Europlanet Science Congress 2020, EPSC2020-1057, doi:10.5194/epsc2020-1057, 2020, [8] Wilkinson, M., et al. (2016) The FAIR Guiding Principles for scientific data management and stewardship. Sci Data 3, 160018, doi:org/10.1038/sdata.2016.18, [9] Raugh et al., (2020), VAs $1^{\text {st }}$ year External Board Review report, available online at https://www.europlanet-society.org/europlanet-2024-ri/europlanet-2024-ri-deliverables/, [10] Brandt, C. H., et a., EGU General Assembly 2020, EGU2020-18839, doi: 10.5194/egusphereegu2020-18839, [11] Luzzi, E., et al. (2020) JGR-Planets, 125, doi:10.1029/2019JE006341, [12] 
Gaddis, L., et al. (1997). An overview of the Integrated Software for Imaging Spectrometers (ISIS), in: Lunar and Planetary Science XXVIII. p. 1997, [13] Beyer, R. A., et al. (2018) Earth and Space Science, 5, 537-548, doi:10.1029/2018EA000409, [14] Manaud et al., (2019) EPSC-DPS Joint Meeting, EPSC Abstracts, Vol. 13, EPSC-DPS2019-1654-1, [15] https://wiki.europlanetgmap.eu/bin/view/Main/Documentation/, [16] https://wiki.europlanet- 